\title{
Stage Ia endometrial carcinoma diagnosed on removal of an IUD
}

Tracey A Kay, BM BS(Hons), DFFP, Senior SHO; Stephen D Keay, MD, MRCOG, Specialist Registrar; Patrick Miller, MRCOG, GP/Gynaecological Practitioner, John N Renninson, MRCOG, Consultant Gynaecologist Department of Obstetrics and Gynaecology, Royal Devon and Exeter Hospital, Exeter, UK.

Correspondence: Tracey A Kay, Specialist Registrar Obstetrics and Gynaecology, Plymouth Hospital NHS Trust, Department of Obstetrics and Gynaecology, Derriford Hospital, Plymouth, PL6 8DU, UK.

(Accepted January $\left.5^{\text {th }}, 2000\right)$

\section{Summary}

This is a case report of a 49-year-old woman who presented with offensive vaginal discharge. Her Lippes loop IUD was removed and discovered to have suspicious material attached. Histology report was of endometrial carcinoma. This is the first report of an endometrial carcinoma being completely removed along with an IUD.

Key words

endometrial carcinoma, IUD

\section{Key message points \\ - This is a case of complete removal of endometrial carcinoma with an IUD. \\ - It is important to send suspicious tissue for histological diagnosis. \\ - There are no reports of IUD use predisposing to endometrial carcinoma.}

\section{Case Report}

A 49 year old woman, para $2+1$, presented to her general practitioner (GP) with a 2 month history of a clear, offensive vaginal discharge 3 years following her menopause. She had not used hormone replacement therapy. Cervical cytology 10 years earlier had shown moderate dyskaryosis leading to colposcopy and large loop excision of the transformation zone for CIN1. Subsequent cytology had been negative. She offered no other gynaecological or medical history of relevance.

Examination revealed a normal appearing lower genital tract with a healthy cervix, but intra-uterine contraceptive device (IUD) threads were noted at the external os. The uterus was mobile and not enlarged. No adenexal masses were palpable. The patient had forgotten that an IUD had been inserted nearly 20 years previously. Bacteriological swabs were taken, but no organism was cultured.

A Lippes loop IUD was removed. The IUD looked abnormal, with mucoid material adherent to it. Unsure of its nature, her GP sent it for histology. The tissue was reported as moderately to poorly differentiated adenocarcinoma (grade II - III).

Following referral to the gynaecological oncology clinic, a hysteroscopy was carried out under general anaesthesia. Operative findings were of a raised area measuring $0.5 \times 0.5$ $\mathrm{cm}$ on the left uterine wall. This was biopsied and endometrial curretings were obtained. Histology was inconclusive, showing blood clots and scanty disintegrated endometrial fragments. The initial pathology specimen from the patient was reviewed in the joint gynaecological oncology/pathology meeting, and the diagnosis was confirmed.

After discussion with the patient a decision was made to proceed with a total abdominal hysterectomy and bilateral salpingo-ophrectomy; this was carried out the following week. The operation was unremarkable and no abnormal masses or lymph nodes were palpated. Histology reported no residual carcinoma of the endometrium, staging her as an Ia cancer of the endometrium. The patient made an uncomplicated postoperative recovery and no further treatment is planned. Follow-up will be in the joint gynaeoncology clinic.

\section{Discussion}

This is the first report of a stage Ia endometrial carcinoma being completely removed along with an IUD, in this case a Lippes loop. It is likely that the lesion was developing as a polyp within the endometrial cavity.

One may suggest that had the IUD not been removed, then the endometrial carcinoma may have progressed. Given the moderate to poorly differentiated nature of the tumour, it would then have had a higher risk of nodal metastasis. If the tissue removed with the IUD had not been examined, the diagnosis would have remained unknown. The subsequent progress in this situation is uncertain, but the patient is likely to have had a higher risk of subsequent carcinoma developing. This emphasises the need to send tissue for histological diagnosis, particularly when the nature of the tissue is uncertain, however it is obtained.

This patient did not have any of the known risk factors for developing endometrial carcinoma (obesity, diabetes, tamoxifen exposure or nulliparity ${ }^{1}$ ) and was younger than the average peak incidence of 61 years of age. ${ }^{2}$ It is unlikely that the IUD predisposed to the endometrial cancer. It is known that IUDs invoke acute and chronic inflammatory responses in the endometrium, but studies have shown that IUD use is not associated with an increased risk of endometrial cancer. ${ }^{3}$ Some data, in fact, suggest a protective role of IUD use on endometrial carcinogenesis. ${ }^{4}$

\footnotetext{
Statements on funding and competing interests

Funding. None declared.

Competing interests. None declared.

References

Canavan TP, Doshi NR. Endometrial Cancer. American Family Physician 1999; 59 (11) $3069-77$

Soutter WP. Malignant disease of the uterus. Dewhurst's textbook of obstetrics and gynaecology for postgraduates. London: Blackwell Science, 2000

Stugeon SR, Brinton LA, Berman ML, et al. Intrauterine device use and Endometrial cance risk. International Journal of Epidemiology 1997; 26(3): 496-500.

4 Parazzini F, La Vecchia C, Moroni S. Intrauterine device use and risk of endometrial cancer British Journal of Cancer 1994; 70 (4): 672-673.
} 